Received: 02.05 .2018

Revised: 27.06 .2018

Accepted: 31.08 .2018

DOI: $10.17804 / 2410-9908.2018 .5 .038-056$

\title{
FINITE ELEMENT MODELING AND ANALYSIS OF THE EFFECTIVE PROPERTIES OF INHOMOGENEOUSLY POLARIZED POROUS PIEZOCERAMIC MATERIAL WITH PARTIAL METALLIZATION OF PORE SURFACES
}

\author{
A. V. Nasedkin*, A. A. Nasedkina, and A. N. Rybyanets \\ Southern Federal University, 105/42 Bolshaya Sadovaya St., Rostov-on-Don, 344006, Russian Federation \\ *Corresponding author. E-mail: nasedkin@math.sfedu.ru \\ Address for correspondence: ul. Miltchakova, 8a, Rostov-on-Don, 344090, Russian Federation \\ Tel.: +7 8632975282
}

The paper considers computational homogenization problems for porous piezoceramic materials with partially metallized pore surfaces. The investigation is based on a complex approach including the effective moduli method, modeling of representative volumes with closed random porosity and metalized pore surfaces, finite element solution of a set of static piezoelectric problems with special boundary conditions and postprocessing of the computation results. Static problems of the piezoelectricity theory for an inhomogeneous representative volume are solved numerically with the help of the ANSYS finite element package. It is assumed that the thickness of the metal layer at the boundaries of the pores is infinitesimally small; therefore, the pore metallization is taken into account only by the electric boundary conditions of equipotentiality on the pore boundaries. Following the previous research, here we simulate the nonuniform polarization field around the pores. The porosity dependences of the effective moduli are analyzed for homogeneous and inhomogeneous polarization fields. The computation results have shown that microporous piezoceramics with metalized pore surfaces has a range of extreme properties promising for practical use.

Keywords: piezoelectricity, porous piezoceramics, microstructure, metallized micropore, effective module, representative volume, finite element method.

\section{Acknowledgements}

This research was supported by grant 16-58-48009 from the Russian Foundation for Basic Research and by grant 9.5070.2017/6.7 of the Russian Ministry of Education and Sciences for the first author.

\section{References}

1. Mercadelli E., Sanson A., Galassi C. Porous piezoelectric ceramics. In: Piezoelectric Ceramics, ed. E. Suaste-Gomez. InTechOpen, 2010, pp. 111-128. DOI: 10.5772/9942.

2. Ringgaard E., Lautzenhiser F., Bierregaard L.M., Zawada T., Molz E. Development of porous piezoceramics for medical and sensor applications. Materials, 2015, vol. 8, no. 12, pp. 8877-8889. DOI: 10.3390/ma8125498.

3. Rybyanets A.N. Porous piezoceramics: theory, technology, and properties. IEEE Trans. Ultrason. Ferroelectr. Freq. Control. 2011, vol. 58, pp. 1492-1507. DOI: 10.1109/TUFFC.2011.1968.

4. Topolov V.Yu., Bowen C.R. Electromechanical Properties in Composites Based on Ferroelectrics. Springer, London, 2009, 202 p. DOI: 10.1007/978-1-84882-000-5.

5. Liu W., Liu W., Wang Y., Xue C., Wang J., Yang J. Piezoelectric and mechanical properties of $\mathrm{CaO}$ reinforced porous PZT ceramics with one-dimensional pore channels. Ceramics International, 2017, vol. 43, pp. 2063-2068. DOI: 10.1016/j.ceramint.2016.10.181. 
6. Wang J., Nie H., Lan C., Wang G., Dong X., Chen X., Cao F., He H. Microstructure, electrical and mechanical properties of $\mathrm{MgO}$ nanoparticles-reinforced porous PZT 95/5 ferroelectric ceramics. Ceramics International, 2013, vol. 39, pp. 3915-3919. DOI: 10.1016/j.ceramint.2012.10.238.

7. Xiang P.-H., Dong X.-L., Chen H., Zhang Z., Guo J.-K. Mechanical and electrical properties of small amount of oxides reinforced PZT ceramics. Ceramics International, 2003, vol. 29, pp. 499-503. DOI: 10.1016/S0272-8842(02)00193-1.

8. Rybyanets A.N., Naumenko A.A. Nanoparticles transport in ceramic matrixes: a novel approach for ceramic matrix composites fabrication. Journal of Modern Physics, 2013, vol. 4, no. 8, pp. 1041-1049. DOI: 10.4236/jmp.2013.48140.

9. $\quad$ OST 11 0444-87 (IUS 3-88). Piezoceramic Materials. Specifications, 1988, 142 p.

10. IEEE Standard on piezoelectricity. ANSI/IEEE Std. 176-1987, New York, IEEE, 1987, 66 p.

11. Bowen C.R., Perry A., Lewis A.C.F., Kara H. Processing and properties of porous piezoelectric materials with high hydrostatic figures of merit. J. Eur. Ceram. Soc., 2004. vol. 24, pp. 541545. DOI: 10.1016/S0955-2219(03)00194-8.

12. Li J.F., Takagi K., Ono, M., Pan W., Watanabe R., Almajid A., Taya M. Fabrication and evaluation of porous piezoelectric ceramics and porosity-graded piezoelectric actuators. J. Am. Ceram. Soc., 2003, vol. 86, pp. 1094-1098. DOI: 10.1111/j.1151-2916.2003.tb03430.x.

13. Liu W., Du L., Wang Y., Yang J., Xu H. Effects of foam composition on the microstructure and piezoelectric properties of macroporous PZT ceramics from ultrastable particle-stabilized foams. Ceramics International, 2013, vol. 39, pp. 8781-8787. DOI: 10.1016/j.ceramint.2013.04.065.

14. Yang A.K., Wang C.A., Guo R., Huang Y. Microstructure and electrical properties of porous PZT ceramics fabricated by different methods. J. Am. Ceram. Soc., 2010, vol. 93, pp. 1984-1990. DOI: 10.1111/j.1551-2916.2010.03684.x.

15. Getman I., Lopatin S. Theoretical and experimental investigation of the porous PZT ceramics. Ferroelectrics, 1996, vol. 186, pp. 301-304. DOI: 10.1080/00150199608218088.

16. Hikita K., Yamada K., Nishioka M., Ono M. Piezoelectric properties of the porous PZT and the porous PZT composite with silicone rubber. Ferroelectrics, 1983, vol. 49, pp. 265-272. DOI: $10.1080 / 00150198308244698$.

17. Bowen C.R., Kara H. Pore anisotropy in 3-3 piezoelectric composites. Materials Chemistry and Physics, 2002, vol. 75, pp. 45-49. DOI: 10.1016/S0254-0584(02)00028-7.

18. Dunn H., Taya M. Micromechanics predictions of the effective electroelastic moduli of piezoelectric composites. Int. J. Solids Struct., 1993, vol. 30, pp. 161-175. DOI: 10.1016/00207683(93)90058-F.

19. Iyer S., Alkhader M., Venkatesh T.A. On the relationships between cellular structure, deformation modes and electromechanical properties of piezoelectric cellular solids. Int. J. Solids Struct., 2016, vol. 80, pp. 73-83. DOI: 10.1016/j.ijsolstr.2015.10.024.

20. Iyer S., Venkatesh T.A. Electromechanical response of (3-0,3-1) particulate, fibrous, and porous piezoelectric composites with anisotropic constituents: A model based on the homogenization method. Int. J. Solids Struct., 2014, vol. 51, pp. 1221-1234. DOI: 10.1016/j.ijsolstr.2013.12.008.

21. Lewis R.W.C., Dent A.C.E., Stevens R., Bowen C.R. Microstructural modelling of the polarization and properties of porous ferroelectrics. Smart Mater. Struct., 2011, vol. 20, pp. 085002. DOI: 10.1088/0964-1726/20/8/085002.

22. Martinez-Ayuso G., Friswell M.I., Adhikari S., Khodaparast H.H., Berger H. Homogenization of porous piezoelectric materials. Int. J. Solids Struct., 2017, vol. 113-114, pp. 218-229. DOI: 10.1016/j.ijsolstr.2017.03.003.

23. Nasedkin A.V., Shevtsova M.S. Simulation of effective moduli for various types of porous piezoceramic materials. Vestnik DGTU, 2013, nos. 3-4 (72-73), pp. 16-26. (In Russian).

24. Nasedkin A.V., Shevtsova M.S. Multiscale computer simulation of piezoelectric devices with elements from porous piezoceramics. In: Physics and mechanics of new materials and their applications, eds. I.A. Parinov and S.-H. Chang, New York, Nova Science Publishers, 2013, ch. 16, pp. 185-202. 
25. Nguyen B.V., Challagulla K.S., Venkatesh T.A., Hadjiloizi D.A., Georgiades A.V. Effects of porosity distribution and porosity volume fraction on the electromechanical properties of 3-3 piezoelectric foams. Smart Mater. Struct., 2016, vol. 25, pp. 125028. DOI: 10.1088/0964$1726 / 25 / 12 / 125028$.

26. Pankov A.A. Statisticheskaya mekhanika piezokompozitov [Statistical Mechanics of Piezocomposites]. Perm, Izd-vo Permskogo Gosudarstvennogo Tekhnicheskogo Universiteta Publ., 2009, 480 p. (In Russian).

27. Perry A., Bowen C.R., Mahon S.W. Finite element modelling of 3-3 piezocomposites. Scripta Materialia, 1999, vol. 41, no. 9, pp. 1001-1007. DOI: 10.1016/S1359-6462(99)00249-3.

28. Sokolkin Yu.V., Pankov A.A. Elektrouprugost piezokompozitov s neregulyarnymi strukturami [Electroelasticity of Piezocomposites with Irregular Structures]. Moscow, Fizmatlit Publ., 2003, 176 p. (In Russian).

29. Khoroshun L.P., Maslov B.P., Leshchenko P.V. Prognozirovanie effektivnykh svoistv piezoaktivnykh kompozitnykh materialov [Prediction of the Effective Properties of Piezoelectric Composite Materials]. Kiev, Naukova Dumka Publ., 1989, 347 p. (In Russian).

30. Nasedkin A.V., Nasedkina A.A., Rybyanets A.N. Finite element analysis of effective properties of microporous piezoceramics with partial metallization of pore surfaces, taken into account the boundary conditions of free electrodes. Fundamentalnye Problemy Radioehlektronnogo Priborostroeniya, 2017, vol. 17, no. 2, pp. 336-339. (In Russian).

31. Nasedkin A.V., Nasedkina A.A., Rybyanets A.N. Models of microporous piezoelectric composites produced by the method of transport of metal-containing microparticles. In: Sovremennye problemy mekhaniki sploshnoy sredy: Proc. XVIII Int. Conf., Rostov-on-Don, 7-10 November, 2016, vol. 2, Rostov-on-Don, Izd-vo YuFU Publ, 2016, pp. 122-126. (In Russian).

32. Nasedkin A.V., Nasedkina A.A., Rybyanets A.N. Numerical study of the effect of pore surface metallization rigidity on the properties of microporous piezoceramics obtained by the metalcontaining particle transport method. Fundamentalnye Problemy Radioelektronnogo Priborostroeniya, 2016, vol. 16, no 2, pp. 58-61. (In Russian).

33. Nasedkin A.V. Models and finite element approximations for interacting nanosized piezoelectric bodies and acoustic medium. In: AIP Conference Proceedings, 2017, vol. 1798, pp. 0201811-7. DOI: $10.1063 / 1.4975643$.

34. Nasedkin A., Nasedkina A., Rybyanets A. Finite element simulation of effective properties of microporous piezoceramic material with metallized pore surfaces. Ferroelectrics, 2017, vol. 508, pp. 100-107. DOI: 10.1080/00150193.2017.1289569.

35. Nasedkin A.V., Nasedkina A.A., Rybyanets A.N. Mathematical modeling and computer design of piezoceramic materials with random arrangement of micropores and metallized pore surfaces. In: Proceedings of the 2016 International Conference on "Physics, Mechanics of New Materials and their Applications”, eds. I.A. Parinov, S.-H. Chang, M.A. Jani. New York, Nova Science Publishers, 2017, ch. 54, pp. 385-392.

36. Nasedkin A.V., Nasedkina A.A., Rybyanets A.N. Modeling and computer design of piezoceramic materials with stochastic microporous structure and local alloying pore surfaces. In: Poromechanics VI: Proceedings of the Sixth Biot Conference on Poromechanics, July 9-13, 2017, Paris, France, eds. M. Vandamme, P. Dangla, J.-M. Pereiram, S. Ghabezloo. Publ. ASCE, Reston, Virginia, USA, 2017, pp. 724-731. DOI: 10.1061/9780784480779.089.

37. Nan C.W., Weng G.J. Influence of polarization orientation on the effective properties of piezoelectric composites. J. Appl. Phys., 2000, vol. 88, no. 1, pp. 416-423. DOI: 10.1063/1.373675.

38. Lekhnitskii S.G. Theory of Elasticity of an Anisotropic Elastic Body, San Francisco, CA, Holden-Day, 1963, 404 p.

39. Nasedkin A.V., Nasedkina A.A., Rajagopal A. Analysis of cymbal transducer from porous piezoceramics PZT-4 with various material properties based on ANSYS. In: Advanced Materials: Proceedings of the International Conference on "Physics and Mechanics of New Materials and Their Applications", PHENMA 2017, Springer Proceedings in Physics, vol. 207, eds. 
I.A. Parinov, S.-H. Chang, V.K. Gupta. Heidelberg, New York, Dordrecht, London, Springer Cham, 2018. DOI: 10.1007/978-3-319-78919-4_42.

40. Wang K.F., Wang B.L., Kitamura T. A review on the application of modified continuum models in modeling and simulation of nanostructures. Acta Mech. Sin., 2016, vol. 32, no. 1, pp. 83-100. DOI: $10.1007 / \mathrm{s} 10409-015-0508-4$. 
Подана в журнал: 02.05.2018

УДК 539.3

DOI: $10.17804 / 2410-9908.2018 .5 .038-056$

\title{
КОНЕЧНО-ЭЛЕМЕНТНОЕ МОДЕЛИРОВАНИЕ И АНАЛИЗ ЭФФЕКТИВНЫХ СВОЙСТВ НЕОДНОРОДНО ПОЛЯРИЗОВАННОГО ПОРИСТОГО ПЬЕЗОКЕРАМИЧЕСКОГО МАТЕРИАЛА С ЧАСТИЧНОЙ МЕТАЛЛИЗАЦИЕЙ ПОВЕРХНОСТЕЙ ПОР
}

\author{
А. В. Наседкин ${ }^{*}$, А. А. Наседкина, А. Н. Рыбянец \\ Южный федеральный университет, \\ ул. Б. Садовая, 105/42, Ростов-на-Дону, Российская Федерация
}

\author{
*Ответственный автор. Электронная почта: nasedkin @ math.sfedu.ru \\ Адрес для переписки: 344090, Ростов-на-Дону, ул. Мильчакова, 8а, Российская Федерация \\ Тел.: +7 (863) 297-52-82
}

В работе рассмотрены задачи гомогенизации для пористого пьезокерамического материала с частично металлизированными поверхностями пор. Предполагается, что толщина слоя металла на границах пор пренебрежимо мала, и эффект металлизации заключается только в задании граничных условий для эквипотенциальных поверхностей. В развитие проведенных ранее исследований здесь учитывается неоднородность поляризации пьезокерамики. Для решения задач гомогенизации использован метод эффективных модулей, метод конечных элементов и конструкции представительных объемов с закрытой случайной пористостью. Проведен анализ зависимостей эффективных модулей от пористости для однородного и неоднородного полей поляризации.

Ключевые слова: пьезоэлектричество, пористая пьезокерамика, микроструктура, металлизированная микропора, эффективный модуль, представительный объем, метод конечных элементов

\section{1. Введение}

В последние годы активно разрабатываются пьезокерамические композиционные материалы и, в частности, пористые пьезокерамические материалы с улучшенными эксплуатационными характеристиками [1-4]. Пористые композитные материалы отличают меньшие плотность и акустический импеданс по сравнению с аналогичными сплошными материалами. Эти свойства обеспечивают преимущества пористых материалов для ряда приложений, например в элементах гидроакустических излучателей. Однако главным недостатком пористых материалов является их меньшая прочность, что ограничивает их применение при больших механических нагрузках. Направленное изменение физико-механических свойств может быть проведено еще на этапе создания пористых материалов [5-8], например, методами локального легирования пор микро- или наночастицами различных веществ. Так, описанная в [8] технология основана на методе транспортировки наночастиц веществ в керамические матрицы. В результате применения данного метода можно получать пористые пьезокерамические материалы, внутри которых на границах керамической матрицы с порами осажены микро- или наночастицы из металла или полимера.

Следует отметить, что исследования эффективных свойств пьезоэлектрических композиционных материалов имеют ряд особенностей по сравнению, например, с исследованиями упругих композитов. Так, пьезоэлектрические материалы обязательно должны быть анизотропными материалами, поскольку, как известно, в силу принципа Пьера Кюри, пьезоэффект не может существовать в материалах с центром симметрии. Кроме того, в силу наличия 
связанности механических и электрических полей, эти материалы характеризуются достаточно большим числом констант. Например, наиболее часто используемые на практике пьезокерамические материалы имеют пять различных модулей жесткости, три пьезомодуля и два коэффициента диэлектрических проницаемостей. Соответственно при решении задач гомогенизации пьезоэлектрических композитных материалов нужно определять такое же число их эффективных модулей (или большее при наличии дополнительной геометрической анизотропии). При использовании экспериментальных методов следует учитывать, что по измеряемым характеристикам можно вычислить материальные модули пьезоэлектрического тела только приближенно, причем часто с достаточно большой погрешностью. Так, для обычной плотной пьезокерамики имеются стандарты определения материальных констант $[9,10]$, базирующиеся на наборах экспериментов с каноническими образцами, для которых математические модели соответствующих процессов описываются простыми, зачастую, одномерными, уравнениями. Понятно, что такие модели являются приближенными, причем их применимость для композитных материалов требует дополнительных обоснований. Возможно, по указанным причинам, а также в связи с большим разнообразим способов получения пьезокерамических композитов, приводимые в литературе экспериментальные данные по материальным модулям пористых пьезокерамических материалов достаточно сильно различаются. При этом имеются различия не только по значениям, но и по тенденциям изменения электромеханических материальных модулей от пористости. Так, в ряде работ [11-14] отмечалось, что эффективный толщинный пьезомодуль $d_{33}^{\text {eff }}$ пористой пьезокерамики убывает с ростом пористости, но другие экспериментальные данные $[3,15,16]$ свидетельствовали о небольшом росте, постоянстве или очень слабом убывании толщинного пьезомодуля при увеличении пористости.

Теоретические и компьютерные исследования пористых пьезоэлектрических материалов, основанные на различных подходах механики композитов [3, 4, 15, 17-29], демонстрируют существенную зависимость значений модулей от структуры пористости, особенно от типа связанности. Естественно, что и используемые модели механики композитов также влияют на получаемые результаты. Среди этих многочисленных подходов в последнее время достаточно часто используется метод эффективных модулей в сочетании с компьютерным моделированием структуры представительного объема и конечно-элементной техникой решения задач гомогенизации $[15,19-25,27]$. Такой подход позволяет в наиболее полной мере учесть внутреннюю структуру пористого композита, включая типы связности, размеры пор и такие локальные эффекты, как неоднородность поля поляризации керамики вблизи пор [21, 23, 24].

Настоящая работа продолжает исследования [30-36] микропористых пьезокомпозитов, внутри которых на границах керамической матрицы с порами осажены микрочастицы из металла. Для численного определения эффективных свойств этих микропористых пьезокерамических материалов использовался подход, включающий метод эффективных модулей, моделирование представительных объемов и конечно-элементное решение набора статических задач теории пьезоэлектричества со специальными граничными условиями.

Методология численного определения эффективных свойств микропористых пьезокерамических материалов с полностью электродированными границами пор была представлена в [31,35] с учетом металлизации граничными условиями свободных электродов и в [32-34] при более общем походе, включающем учет механических свойств металлизированных границ пор посредством оболочечных элементов. Между тем более соответствующими реальности являются модели с частичной металлизацией границ пор. Такой вариант композита рассматривался в $[30,36]$. В настоящей работе, как и в $[30,36]$, металлизация пор моделировалась только условиями свободных электродов, а вся технология расчета эффективных свойств была реализована в конечно-элементном комплексе ANSYS. Следует также отметить, что ранее в [30-36] рассматривалась только однородно поляризованная пьезокерамика, несмотря на наличие в ней пор и металлизированных поверхностей. Здесь, аналогично 
$[23,24]$, проведено исследование влияния на эффективные модули неоднородности поля поляризации, что существенно отличает настоящую работу от $[30,36]$.

\section{2. Математические модели и метод эффективных модулей}

Рассмотрим неоднородно поляризованный пористый пьезоэлектрический композит с границами пор, частично покрытыми очень тонким слоем металла. Для общности будем считать пористый композит двухфазным, в котором первая фаза или матрица композита является пьезокерамическим материалом с неоднородной поляризацией, а вторая фаза представляет собой совокупность пор, не соприкасающихся друг с другом.

Пусть $\mathbf{x}$ - радиус-вектор точки в декартовой системе координат; $\Omega$ - представительный объем композита; $\Gamma=\partial \Omega-$ внешняя граница объема. Будем считать, что в объеме имеются две фазы композита $\Omega=\Omega_{m} \cup \Omega_{p} ; \Omega_{m}$ - область, занимаемая материалом первой фазы или матрицей; $\Omega_{p}$ - область второй фазой, занимаемая порами. При закрытой пористости примем, что $\Omega_{p}$ является объединением $N_{p}$ не соприкасающихся между собой пор $\Omega_{p i}$; $\Omega_{p}=\cup_{i=1}^{N_{p}} \Omega_{p i} ; \Gamma_{p i}=\partial \Omega_{p i}-$ границы пор, $i=1,2, \ldots, N_{p}$. Обозначим через $\mathbf{n}-$ вектор единичной нормали к границе $\Gamma_{m}=\partial \Omega_{m}$, внешней по отношению к объему основного материала. Внутри каждой пористой границы $\Gamma_{p i}$ выделим металлизированные участки $\Gamma_{p i j}^{e}$, $j=1,2, \ldots, J_{i}^{e} \quad$ и неметаллизированные участки $\Gamma_{p i j}^{u}, j=1,2, \ldots, J_{i}^{u}$. Таким образом, $\Gamma_{p i}=\left(\cup_{j} \Gamma_{p i j}^{e}\right) \cup\left(\cup_{j} \Gamma_{p i j}^{u}\right)$, причем участки $\Gamma_{p i j}^{e}$ не касаются друг друга. Металлизированные поверхности керамики часто называются электродированными, а неметаллизированные поверхности - неэлектродированными или участками, свободными от электродов.

Для определения эффективных модулей композита рассмотрим следующие статические краевые задачи теории упругости [30-36]:

$$
\begin{gathered}
\mathbf{L}^{*}(\nabla) \cdot \mathbf{T}=0 ; \nabla \cdot \mathbf{D}=0 ; \\
\mathbf{T}=\mathbf{c}^{E} \cdot \mathbf{S}-\mathbf{e}^{*} \cdot \mathbf{E} ; \quad \mathbf{D}=\mathbf{e} \cdot \mathbf{S}+\boldsymbol{\varepsilon}^{S} \cdot \mathbf{E} ; \\
\mathbf{S}=\mathbf{L}(\nabla) \cdot \mathbf{u} ; \quad \mathbf{E}=-\nabla \varphi ; \\
\mathbf{u}=\mathbf{L}^{*}(\mathbf{x}) \cdot \mathbf{S}_{0} ; \varphi=-\mathbf{x} \cdot \mathbf{E}_{0} ; \quad \mathbf{x} \in \Gamma
\end{gathered}
$$

Здесь $\mathbf{T}=\left\{\sigma_{11}, \sigma_{22}, \sigma_{33}, \sigma_{23}, \sigma_{13}, \sigma_{12}\right\} ; \mathbf{S}=\left\{\varepsilon_{11}, \varepsilon_{22}, \varepsilon_{33}, 2 \varepsilon_{23}, 2 \varepsilon_{13}, 2 \varepsilon_{12}\right\} ; \sigma_{i j}, \varepsilon_{i j}-$ компоненты тензоров напряжений и деформаций; $\mathbf{D}, \mathbf{E}-$ векторы электрической индукции и напряженности электрического поля соответственно; $\mathbf{u}=\mathbf{u}(\mathbf{x})$ - вектор перемещений; $\varphi=\varphi(\mathbf{x})$ - электрический потенциал; $\mathbf{c}^{E}$ - матрица упругих жесткостей размера $6 \times 6$, измеренных при постоянном электрическом поле; е - матрица пьезомодулей размера $3 \times 6 ; \boldsymbol{\varepsilon}^{S}$ - матрица диэлектрических проницаемостей размера $3 \times 3$, измеренных при постоянных деформациях; $\mathbf{S}_{0}=\left\{S_{01}, S_{02}, S_{03}, S_{04}, S_{05}, S_{06}\right\} ; S_{0 \alpha}$ - некоторые постоянные значения, не зависящие от $\mathbf{x}$; $\mathbf{E}_{0}$ - некоторый постоянный вектор; (...) ${ }^{*}$ - операция транспонирования; (...) $\cdot(\ldots)$ - операция 
скалярного или внутреннего произведения; $\nabla=\left\{\partial_{1}, \partial_{2}, \partial_{3}\right\}$ - набла-оператор; $\mathbf{L}(\mathbf{a})$ - матричный оператор размера $6 \times 3$ с векторным аргументом $\mathbf{a}, \mathbf{L}^{*}(\mathbf{a})=\left[\begin{array}{cccccc}a_{1} & 0 & 0 & 0 & a_{3} & a_{2} \\ 0 & a_{2} & 0 & a_{3} & 0 & a_{1} \\ 0 & 0 & a_{3} & a_{2} & a_{1} & 0\end{array}\right]$.

Отметим, что задача (1)-(4) решается в объеме $\Omega$ с кусочно-однородными материальными свойствами: $\mathbf{c}^{E}=\mathbf{c}^{E(r)}, \mathbf{e}=\mathbf{e}^{(r)}, \boldsymbol{\varepsilon}^{S}=\boldsymbol{\varepsilon}^{S(r)}$ для $\mathbf{x} \in \Omega_{r}, r=m, p$. Предполагается, что поры заполнены пьезоэлектрическим материалом с пренебрежимо малыми упругими жесткостями, прьезомодулями и диэлектрическими проницаемостями, равными диэлектрической проницаемости вакуума $\varepsilon_{0}=8.85 \cdot 10^{-12}(\Phi / \mathrm{M})$.

Для обычной пористой керамики без металлизации на границах пор выполняются условия отсутствия механических напряжений и поверхностных электрических зарядов:

$$
\mathbf{L}^{*}(\mathbf{n}) \cdot \mathbf{T}=0 ; \mathbf{n} \cdot \mathbf{D}=0 ; \quad \mathbf{x} \in \Gamma_{p i} .
$$

В принятой модели, когда поры считаются заполненными пьезоэлектрическим материалом с малыми модулями, условия (5) выполняются приближенно, но с большой точностью.

Между тем, когда границы пор частично покрыты металлом, причем его толщина пренебрежимо мала, то условия (5) сохраняются на участках $\Gamma_{p i j}^{u}$, но на металлизированных участках $\Gamma_{p i j}^{e}$ следует принять граничные условия свободных электродов. В итоге вместо (5) надо использовать следующие граничные условия:

$$
\begin{gathered}
\mathbf{L}^{*}(\mathbf{n}) \cdot \mathbf{T}=0 ; \quad \mathbf{x} \in \Gamma_{p i} ; \\
\mathbf{n} \cdot \mathbf{D}=0 ; \quad \mathbf{x} \in \Gamma_{p i j}^{u} ; \quad j=1,2, \ldots, J_{i}^{u} ; \\
\varphi=\Phi_{i j} ; \quad \quad \mathbf{x} \in \Gamma_{p i j}^{e} ; \quad \int_{\Gamma_{p i j}^{e}} \mathbf{n} \cdot \mathbf{D} d \Gamma=0 \quad j=1,2, \ldots, J_{i}^{e},
\end{gathered}
$$

где $\Phi_{i j}$ - постоянные неизвестные электрические потенциалы на $\Gamma_{p i j}^{e}$.

Пористая пьезокерамика, равномерно поляризованная вдоль оси $O x_{3}$, как известно, является трансверсально-изотропным материалом $6 \mathrm{~mm}$ класса. Поэтому логично предполагать, что и гомогенизация пористого пьезокерамического композита со случайной пористостью и со случайным расположением металлизированных границ пор сохранит тот же класс анизотропии. В этом случае надо определить десять различных эффективных модулей, например: пять эффективных модулей жесткости $c_{11}^{E \text { eff }}, c_{12}^{E \text { eff }}, c_{13}^{E \text { eff }}, c_{33}^{E \text { eff }}, c_{44}^{E \text { eff }}$, три эффективных пьезомодуля $e_{31}^{\text {eff }}, e_{33}^{\text {eff }}, e_{15}^{\text {eff }}$ и два эффективных коэффициента диэлектрических проницаемостей $\varepsilon_{11}^{S \text { eff }}, \varepsilon_{33}^{S \text { eff }}$. Тогда, для вычисления полного набора эффективных модулей композита требуется решить пять задач (1)-(5) или (1)-(4), (6)-(8) с различными значениями $\mathbf{S}_{0}$ и $\mathbf{E}_{0}$, только одна из компонент $S_{0 \alpha}$, или $E_{0 k}$ в граничных условиях (4) отлична от нуля:

$$
\begin{aligned}
& \text { I } \quad S_{0 \alpha}=S_{0} \delta_{1 \alpha}, \mathbf{E}_{0}=0 \Rightarrow c_{1 l}^{E \text { eff }}=\left\langle\sigma_{l l}\right\rangle / S_{0} ; l=1,2,3 ; e_{31}^{\text {eff }}=\left\langle D_{3}\right\rangle / S_{0} ; \\
& \text { II } S_{0 \alpha}=S_{0} \delta_{3 \alpha}, \mathbf{E}_{0}=0 \Rightarrow c_{l 3}^{E \text { eff }}=\left\langle\sigma_{l l}\right\rangle / S_{0} ; l=1,2,3 ; e_{33}^{\text {eff }}=\left\langle D_{3}\right\rangle / S_{0} ;
\end{aligned}
$$




$$
\begin{aligned}
& \text { III } S_{0 \alpha}=S_{0} \delta_{4 \alpha}, \mathbf{E}_{0}=0 \Rightarrow c_{44}^{E \text { eff }}=\left\langle\sigma_{23}\right\rangle / S_{0} ; e_{15}^{\text {eff }}=\left\langle D_{2}\right\rangle / S_{0} ; \\
& \text { IV } \quad \mathbf{S}_{0}=0, E_{0 k}=E_{0} \delta_{1 k} \Rightarrow e_{15}^{\text {eff }}=-\left\langle\sigma_{13}\right\rangle / E_{0} ; \varepsilon_{11}^{S \text { eff }}=\left\langle D_{1}\right\rangle / E_{0} ; \\
& \text { V } \quad \mathbf{S}_{0}=0, E_{0 k}=E_{0} \delta_{3 k} \Rightarrow e_{3 l}^{\text {eff }}=-\left\langle\sigma_{l l}\right\rangle / E_{0} ; l=1,2,3 ; \varepsilon_{33}^{S \text { eff }}=\left\langle D_{3}\right\rangle / E_{0},
\end{aligned}
$$

где $\alpha=1,2, \ldots, 6 ; k=1,2,3 ; \delta_{i j}$ - символ Кронекера; угловые скобки означают осреднения соответствующих величин по представительному объему: $<(\ldots)>=(1 /|\Omega|) \int_{\Omega}(\ldots) d \Omega$.

Задачи I-V, т.е. задачи (1)-(5) или (1)-(4), (6)-(8) с (9)-(13) будем решать численно по методу конечных элементов (МКЭ) в неоднородном представительном объеме $\Omega$, предварительно моделируя внутреннюю микроструктуру композита.

\section{3. Моделирование представительных объемов}

Представительный объем $\Omega$ пористого композита с закрытой пористостью будем строить в виде конечно-элементного массива, в котором конечные элементы первой фазы композита будут иметь материальные свойства основного материала, а элементы второй фазы - материальные свойства пор. В основу построения структуры объема $\Omega$ положим базовую кубическую ячейку $\Omega_{c}$ с ребром $l_{c}$. Ячейку $\Omega_{c}$ разобьем по каждой стороне на три части с длинами $a_{p}, l_{p}, a_{p}$, где $a_{p}=\left(l_{c}-l_{p}\right) / 2 ; l_{p}=k_{p} l_{c} ; k_{p}<1$. Таким образом, базовая ячейка $\Omega_{c}$ будет разбита на $3^{3}=27$ прямоугольных параллелепипедов, которые первоначально наделяются материальными свойствами диэлектрических конечных элементов. По центру базовой ячейки располагается основной элемент в форме куба со стороной $l_{p}$. Общий массив конечных элементов $\Omega$ получим в результате копирования базовой ячейки по трем координатным осям по $n_{c}$ раз. В итоге массив $\Omega$ будет состоять из $n_{c}^{3}$ базовых ячеек и иметь форму куба со стороной $L=n_{c} l_{c}$.

Будем считать, что порами могут быть только центральные конечные элементы внутри базовых ячеек. Эти пористые элементы выберем случайным образом, задав на вход алгоритма предполагаемую пористость $p_{s}$, как отношение желаемого объема пор к общему объему. Предполагаемая пористость тогда будет незначительно отличаться от истинной пористости $p$, которая вычисляется по формуле $p=N_{p} l_{p}^{3} / L^{3}=N_{p}\left(k_{p} / n_{c}\right)^{3}$. Здесь $N_{p}$ - число центральных конечных элементов, которые могут быть порами, определяемое по формуле: $N_{p}=\left[p_{s}\left(n_{c} / k_{p}\right)^{3}\right]$, где [...] - целая часть числа. Эти $N_{p}$ кубических элементов выберем датчиком случайных чисел, и после выбора их материальные свойства модифицируем на свойства пор.

Для моделирования частичной металлизации среди шести граней пористого конечного элемента электродированными будем считать только две противоположные грани, расположенные перпендикулярно одному из осевых направлений $x_{k}$. При этом, направление $x_{k}$ для каждого пористого элемента выбирается случайным образом между направлениями осей координат $x_{1}, x_{2}, x_{3}$. Тогда в представительном объеме $\Omega$ будет содержаться $N_{p}$ пористых элементов $\Omega_{p i}, i=1,2, \ldots, N_{p}$, У которых суммарно $2 N_{p}$ граней $\Gamma_{p i j}^{e}$ электродированы, причем эти парные грани $(j=1,2)$ будут ориентированы случайным образом вдоль координатных осей. 


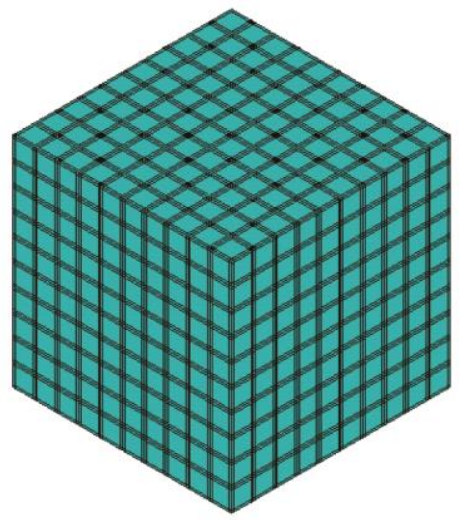

$a$

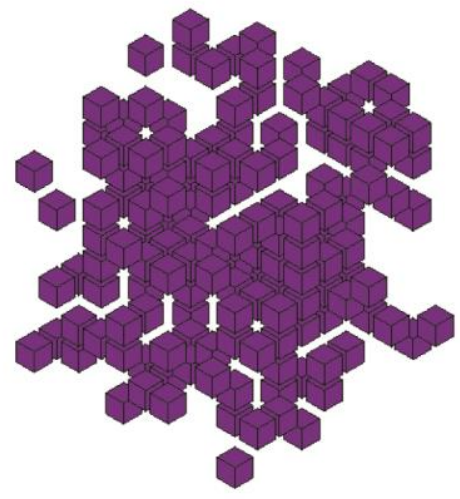

$\sigma$

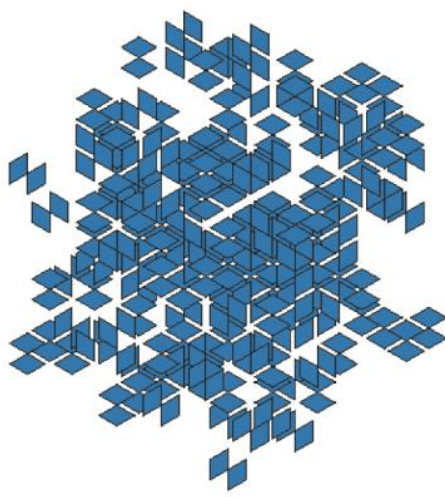

B

Рис. 1. Пример представительного объема: $а$ весь объем; б элементы - поры; в поверхности пор с металлизацией

На рис. $1(a-8)$ показан один из вариантов объема $\Omega$, построенного по описанному алгоритму при $p_{s}=0,1, n_{c}=10, k_{p}=0,8$. Отметим, что «пористые» элементы $\Omega_{p i}$, показанные на рис. 1, б, выбираются среди центральных элементов базовых ячеек датчиком случайных чисел и поэтому при следующем запуске алгоритма их расположение поменяется. Не является детерминированным также выбор металлизированных поверхностей, показанных на рис. 1, в. Поэтому расположение генерируемых поверхностей $\Gamma_{p i}$ также будет изменяться при следующем проходе алгоритма, причем даже в том случае, если пористые элементы случайно останутся прежними.

В результате получается представительный объем пористого материала с закрытой пористостью частично стохастической структуры. В этом объеме имеется $N_{p}$ элементов-пор $\Omega_{p i}$, все грани которых полностью контактируют с границами соседствующих элементов матрицы композитного материала. При этом в каждой поре по две противоположные грани $\Gamma_{p i j}^{e}$ выбраны металлизированными.

\section{4. Моделирование неоднородной поляризации и стратегия конечно-элементного решения}

Как известно, пьезокерамика является трансверсально-изотропным материалом класса $6 m m$. Стандартно она принимается однородно поляризованной в направлении оси $O x_{3}$. Поляризация пьезокерамического образца осуществляется технологически, для чего необходимо наличие электродов, через которые подается сильное электрическое поле, превосходящее коэрцитивное. Таким образом, поляризация определяется не только материалом, но и геометрией устройства, а также расположением технологических электродов. Пористая пьезокерамика на микроуровне, естественно, является неоднородным материалом. В частности, поле поляризации неоднородно в окрестности пор. Между тем в большинстве исследований, например в [3, 4, 15, 17-20. 22, 25-29], эффективные свойства пористой пьезокрамики определялись в предположении ее однородной поляризации. В $[21,23,24,37]$ исследовалось и влияние неоднородности поля поляризации. Как было показано в $[23,24]$, для малой и средней пористости это влияние достаточно мало.

В случае пористой пьезокерамики с металлизированными поверхностями пор учет неоднородности поля поляризации может быть более существенным. В реальности металлизация пор происходит в процессе спекания пьезокерамики, а поляризация материала осуществляется уже после ее спекания. Понятно, что тогда наличие проводящих поверхностей внутри материала будет оказывать дополнительное влияние на поле поляризации. В связи с этим для учета 
неоднородной поляризации пьезокерамики в окрестности пор на предварительном этапе можно смоделировать процесс поляризации пористой керамики вдоль оси $O x_{3}$. Для этого решим по МКЭ задачу квазиэлектростатики для пористого диэлектрика в представительном объеме $\Omega$, сгенерированном по методу, описанному в предыдущем разделе.

Именно для неоднородного куба $\Omega$ со стороной $L$ рассмотрим в декартовой системе координат $O x_{1} x_{2} x_{3}$ следующую краевую задачу

$$
\begin{gathered}
\nabla \cdot \mathbf{D}=0 ; \mathbf{D}=\varepsilon \cdot \mathbf{E} ; \mathbf{E}=-\nabla \varphi ; \quad \mathbf{x} \in \Omega, \\
\varphi=V_{j} ; \quad \mathbf{x} \in \Gamma_{\varphi_{j}} ; j=1,2 ; \quad \mathbf{n} \cdot \mathbf{D}=0, \mathbf{x} \in \Gamma_{q},
\end{gathered}
$$

где $\Gamma=\Gamma_{\varphi 1} \cup \Gamma_{\varphi 2} \cup \Gamma_{q} ; \Gamma_{\varphi 1}, \Gamma_{\varphi 2}$ - электродированные грани $x_{3}=0$ и $x_{3}=L$, на которые подаются различные значения $V_{1}$ и $V_{2}$ электрического потенциала соответственно. Разность электрических потенциалов $V_{1}$ и $V_{2}$ определяют величину подаваемого вдоль оси $O x_{3}$ поля поляризации $E_{p}=\left(V_{1}-V_{2}\right) / L$. Наконец, $\boldsymbol{\varepsilon}=\boldsymbol{\varepsilon}(\mathbf{x})-$ диагональная матрица диэлектрических проницаемостей неполяризованной керамики с порами, которая в объеме $\Omega$ считается изотропным материалом с кусочно-однородными свойствами $\boldsymbol{\varepsilon}=\boldsymbol{\varepsilon}^{(r)} ; r=m, p$, причем $\varepsilon_{k k}^{m}=\left(2 \varepsilon_{11}^{S}+\varepsilon_{31}^{S}\right) / 3 ; \varepsilon_{k k}^{p}=\varepsilon_{0} ; k=1,2,3$.

К задаче (14), (15) надо еще добавить электрические граничные условия (7), (8), действующие на поверхностях пор. После решения сформулированной задачи по МКЭ можно найти значения векторов поляризации $\mathbf{P}^{e k}=\mathbf{D}^{e k}-\varepsilon_{0} \mathbf{E}^{e k}$ в центре каждого конечного элемента с номером $k$, не являющегося порой. С данными элементами ассоциировались свои элементные системы координат $O x_{1}^{e k} x_{2}^{e k} x_{3}^{e k}$, для которых оси $O x_{3}^{e k}$ выбирали такими, чтобы их направления совпадали с направлениями векторов поляризации $\mathbf{P}^{e k}$.

На следующем этапе конечные элементы электростатики были модифицированы в элементы с возможностями пьезоэлектрического анализа. В процессе модификации элементам присваивались материальные свойства двух типов: поляризованной пьезокерамики для элементов матрицы материала и пренебрежимо малые модули для элементов со свойствами пор. При этом с конечными элементами матрицы связывались элементные системы координат $O x_{1}^{e k} x_{2}^{e k} x_{3}^{e k}$, определенные по векторам поляризации $\mathbf{P}^{e k}$.

Далее для определения эффективных модулей решались задачи электроупругости (1)(5) для обычной пористой пьезокерамики или задачи (1)-(4), (6)-(8) для пористой пьезокерамики с частично металлизированными границами пор. Эти задачи решались по пять раз в соответствии с вариантами (9)-(13). Подчеркнем, что при учете неоднородной поляризации задачи электроупругости решались для неоднородной структуры представительного объема, в которой каждый конечный элемент поляризованной пьезокерамики имел свои модули $\mathbf{c}^{E e k}$, $\mathbf{e}^{e k}, \boldsymbol{\varepsilon}^{\text {Sek}}$, получаемые по известным формулам [38] пересчета тензорных коэффициентов при переходе от кристаллографической декартовой системы координат $O x_{1}^{e k} x_{2}^{e k} x_{3}^{e k}$ в элементные системы координат $O x_{1}^{e k} x_{2}^{e k} x_{3}^{e k}$.

Если не учитывать неоднородность поляризации в окрестностях пор, то задача (14), (15) не используется и в задачах (1)-(5) или (1)-(4), (6)-(8) все конечные элементы имеют или свойства пьезокерамического материала класса $6 \mathrm{~mm}$, поляризованного вдоль оси $O x_{3}$, или свойства пор. 


\section{5. Численные результаты}

Решение задач гомогенизации проводилось по методу конечных элементов в программном комплексе ANSYS по методологии, описанной выше. На языке APDL ANSYS были составлены программы, генерирующие представительный объем, решающие вначале задачу электростатики (14), (15), а затем пять задач гомогенизации (1)-(5) или (1)-(4), (6)-(8) с различными граничными условиями (9)-(13). После решений в ANSYS автоматически вычислялись осредненные характеристики и в итоге определялся полный набор эффективных модулей. При расчетах использовался восьмиузловой конечный элемент SOLID5 со степенями свободы перемещений и электрического потенециала, причем для задачи электростатики выбиралась опция со степенью свободы электрического потенециала.

В качестве примера рассмотрим пористую пьезокерамику с матрицей из сегнетомягкого материала PZT-5Н. Для плотной пьезокерамики PZT-5Н возьмем следующие значения материальных констант [23]: $c_{11}^{E}=127,2 ; c_{12}^{E}=80,2 ; c_{13}^{E}=84,7 ; c_{33}^{E}=117,4 ; c_{44}^{E}=22,98$ (ГПа); $e_{33}=23,24 ; e_{31}=-6,62 ; e_{15}=17,03\left(\right.$ Кл $\left./ \mathrm{M}^{2}\right) ; \varepsilon_{11}^{S}=1704,4 \varepsilon_{0} ; \varepsilon_{33}^{s}=1433,6 \varepsilon_{0}$. Для пор зададим пренебрежимо малые упругие модули $c_{\alpha \beta}^{E p}=\kappa c_{\alpha \beta}^{E} ; \kappa=10^{-10}$, пьезомодули $e_{i \alpha}^{p}=\kappa\left(\mathrm{x} 1\right.$ Кл/м $\left.{ }^{2}\right)$ и $\varepsilon_{i i}^{S p}=\varepsilon_{0}$. Для представительного объема примем следующие геометрические параметры: $L=500$ (мкм); $n_{c}=10 ; k_{p}=0,8$. В этом случае поры будут иметь ребра $l_{p}=k_{p} L / n_{c}=40$ (мкм).

Отметим, что при выбранном числе базовых ячеек $n_{c}=10$ представительный объем состоит из $27 n_{c}^{3}=27000$ конечных элементов. Такая сетка содержит 29791 узлов. Для задач пьезоэлектричества в каждом узле имеем 4 степени свободы (три компоненты вектора перемещений и электрический потенциал). Тогда после удовлетворения главных граничных условий (4) и условий эквипотенциальности (8) при решении задач (1)-(4), (6)-(8) с (9)-(13) по методу конечных элементов каждый раз будет решаться система линейных алгебраических уравнений из 91694 уравнений. Большее число базовых ячеек потребует и более существенных компьютерных ресурсов. Например, для $n_{c}=15$ представительный объем будет состоять из 91125 конечных элементов с 97336 узлами, а системы конечно-элементных уравнений задач (1)-(4), (6)-(8) с (9)-(13) будут включать 340660 уравнений. Между тем, вычислительные эксперименты для двух вариантов представительных объемов с $n_{c}=10$ и с $n_{c}=15$ показали, что относительные разности между значениями соответствующих эффективных модулей для пористой пьезокерамики с учетом неоднородной поляризации и частичной металлизации поверхности пор при пористости от 10 до 50 \% не превышали $2 \%$ за исключением значений эффективного пьезомодуля $e_{31}^{\text {eff }}$, где максимальная относительная разность между значениями составила 3,7 \%. Поскольку такая погрешность для модулей пористой пьезокерамики со стохастической пористостью не является слишком большой, то для всех рассматриваемых далее случаев был выбран вариант представительного объема с $n_{c}=10$. При этом поскольку алгоритм создания представительного объема включал работу датчика случайных чисел при генерировании пор и металлизации их границ, то была проведена оценка относительных разностей значений эффективных модулей при трех последовательных работах программы для пористой пьезокерамики с учетом неоднородной поляризации и частичной металлизации поверхности пор при пористости $10 \%\left(p_{s}=0,1\right)$ и $50 \%\left(p_{s}=0,5\right)$. Относительные разности между значениями соответствующих эффективных модулей здесь также не превышали $2 \%$, за исключением значений эффективного пьезомодуля $e_{31}^{\text {eff }}$, где максимальная относительная разность между значениями составила $5,7 \%$ при $p_{s}=0,1$. Такая погрешность также была принята приемлемой и поэтому при анализе получаемых значений эффективных 
модулей использовались результаты вычислений при однократных запусках программ для различных рассматриваемых далее типов пористой пьезокерамики.

Перейдем к описанию полученных результатов, сравнивая два варианта модели пористой пьезокерамики. В варианте 1 учтем металлизацию пор граничными условиями (6)-(8). В варианте 2 рассмотрим обычную пористую пьезокерамику, когда на границах пор нет условий эквипотенциальности, а выполняются только условия (5). Для каждого варианта будем рассматривать случай однородно поляризованной пьезокерамики и случай неоднородно поляризованной пьезокерамики. Проанализируем относительные эффективные модули. Например, $r\left(c_{\alpha \beta}^{E}\right)=c_{\alpha \beta}^{E \text { eff }} / c_{\alpha \beta}^{E}-$ значения эффективных модулей $c_{\alpha \beta}^{E \text { eff }}$, отнесенные к соответствующим значениям модулей $c_{\alpha \beta}^{E}$ сплошной пьезокерамики, и т. д. Также будем использовать индекс $l=1,2$ в уточненных обозначениях $r\left(c_{\alpha \beta}^{E}\right)_{l}=\left(c_{\alpha \beta}^{E \text { eff }}\right)_{l} / c_{\alpha \beta}^{E}$ для указания номера варианта $l$, для которого проводился расчет модулей.

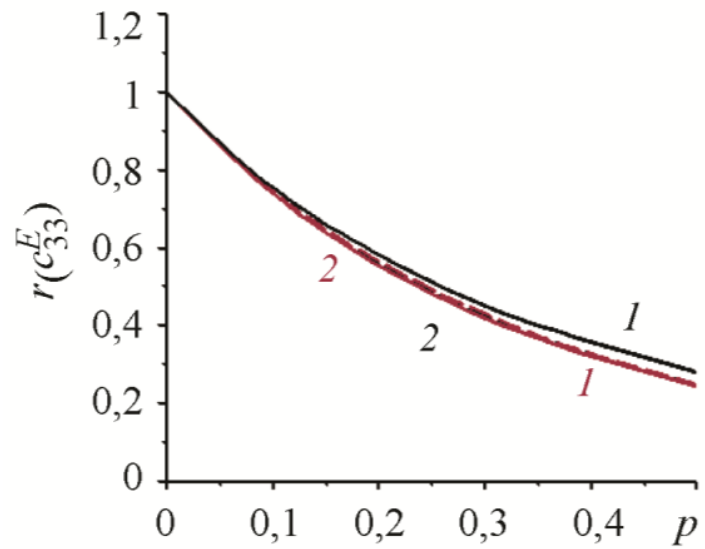

$a$

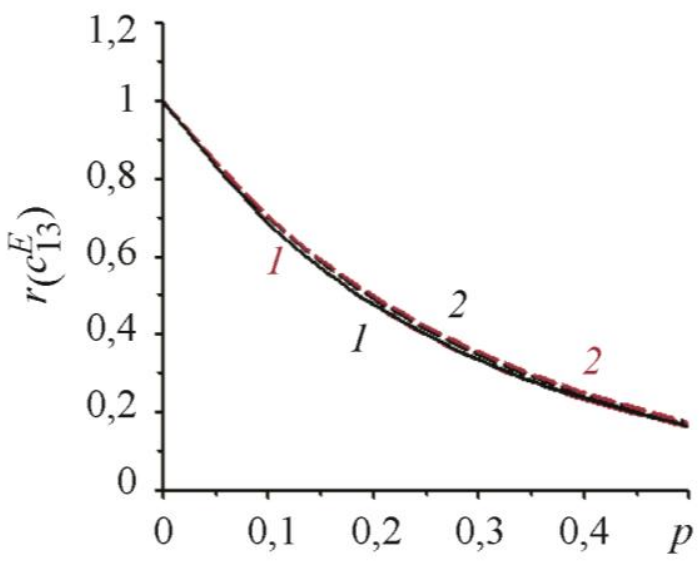

$\sigma$

Рис. 2. Зависимости эффективных упругих жесткостей от пористости

Типичное поведение эффективных модулей жесткости показано на рис. 2 на примере модулей $\left(c_{33}^{E \text { eff }}\right)_{l}$ и $\left(c_{13}^{E \text { eff }}\right)_{l}$, а на рис. 3 показано поведение эффективных модулей диэлектрических проницаемостей $\left(\varepsilon_{11}^{S \text { eff }}\right)_{l}$ и $\left(\varepsilon_{33}^{S \text { eff }}\right)_{l}, l=1,2$. Здесь и далее на рисунках кривые черного цвета соответствуют случаю неоднородно поляризованной пьезокерамики, а кривые коричневого цвета соответствуют случаю пьезокерамики с однородной поляризацией.

Как видно из рис. 2, модули жесткости убывают с ростом пористости для обоих вариантов, причем учет неоднородности поля поляризации достаточно слабо влияет на значения модулей жесткости. Между тем (рис. 3) эффективные модули диэлектрических проницаемостей обычной пористой пьезокерамики убывают с ростом пористости (кривые 2), но эффективные модули диэлектрических проницаемостей пористой пьезокерамики с металлизированными порами, наоборот, возрастают с ростом пористости (кривые 1), причем для модуля $\left(\varepsilon_{33}^{\text {Seff }}\right)_{1}$ этот эффект наблюдается до $p=0,4$. Учет неоднородности поля поляризации слабо влияет на значения модулей диэлектрических проницаемостей $\left(\varepsilon_{11}^{\text {Seff }}\right)_{l}, l=1,2$ и $\left(\varepsilon_{33}^{\text {Seff }}\right)_{2}$. Для модуля $\left(\varepsilon_{33}^{S \text { eff }}\right)_{1}$ пористой пьезокерамики с частично металлизированными границами пор учет неоднородности поля поляризации более существенен и приводит к более сильному изменению диэлектрической проницаемости. 

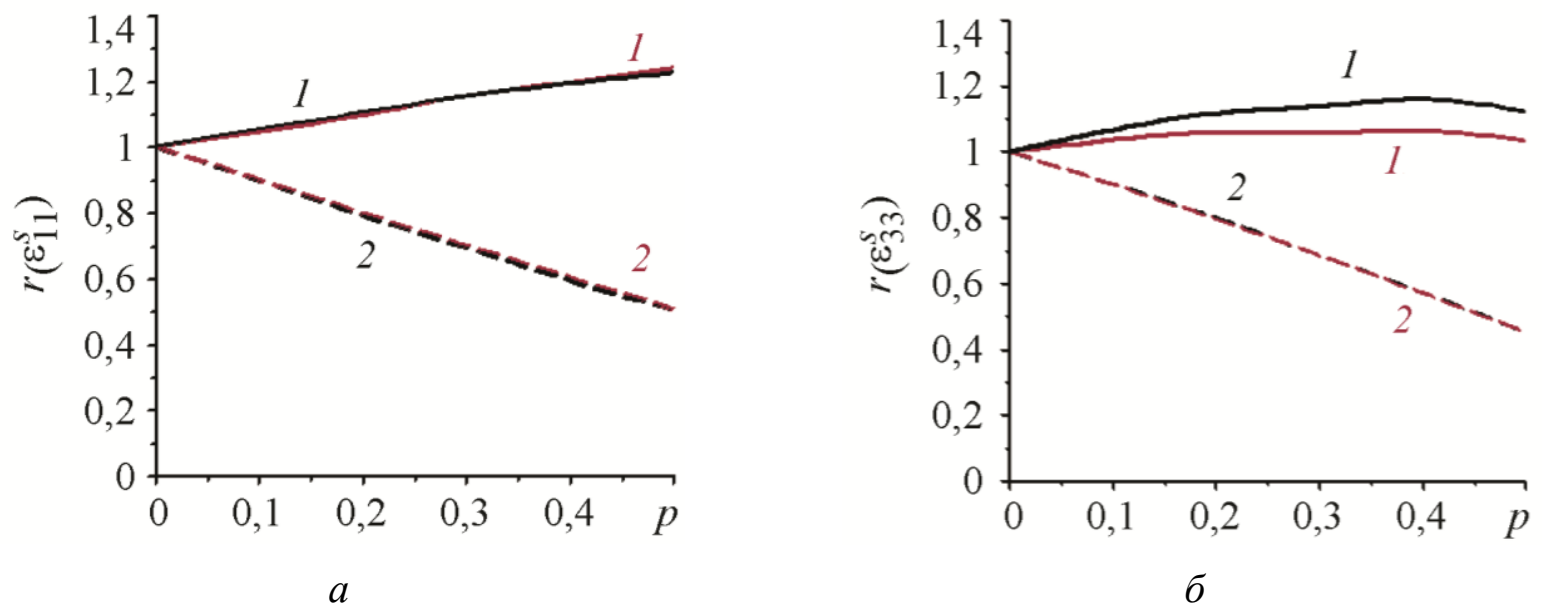

Рис. 3. Зависимости эффективных диэлектрических проницаемостей от пористости

Более интересно поведение пьезомодулей (рис. 4 и 5). Так, пьезомодули $\left(e_{33}^{\text {eff }}\right)_{2}$ и $\left(\left|e_{31}^{\text {eff }}\right|\right)_{2}$ для обычной пористой пьезокерамики убывают с ростом пористости. Между тем, для пьезокерамики с металлизированными поверхностями пор пьезомодуль $\left(e_{33}^{\text {eff }}\right)_{1}$ также убывает с ростом $p$, причем более быстро, чем $\left(e_{33}^{\text {eff }}\right)_{2}$, в то время как пьезомодуль $\left(e_{31}^{\text {eff }}\right)_{1}$ по абсолютной величине, наоборот, возрастает при увеличении пористости до $p=0,4$, а затем стабилизируется или слабо убывает. Учет неоднородности поля поляризации для пьезомодуля $e_{33}^{\text {eff }}$ практически не существенен, а для абсолютной величины пьезомодуля $e_{31}^{\text {eff }}$ дает немного большее убывание для обычной пористой пьезокерамики и немного большее возрастание для пористой пьезокерамики с частичной металлизацией поверхностей пор.

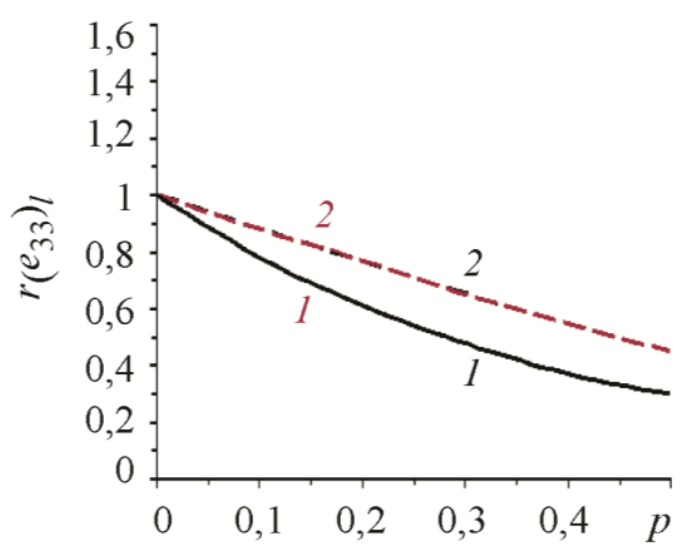

$a$

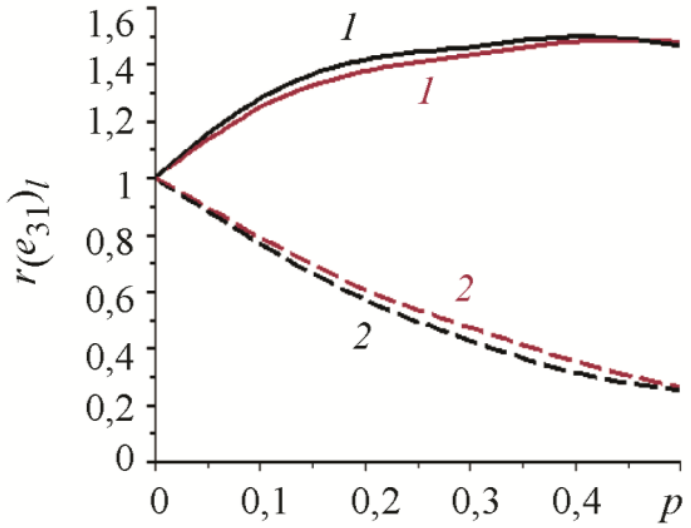

б

Рис. 4. Зависимости эффективных пьезомодулей $r\left(e_{33}\right)_{l}(a)$ и $r\left(e_{31}\right)_{l}(б)$ от пористости 


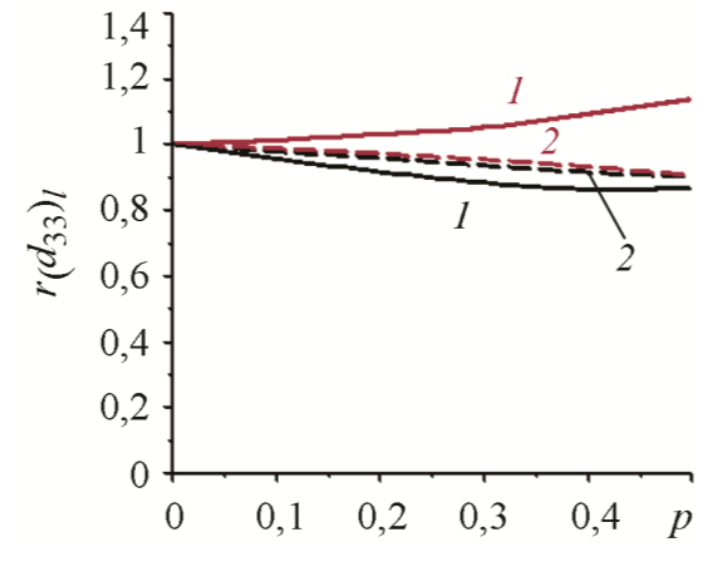

$a$

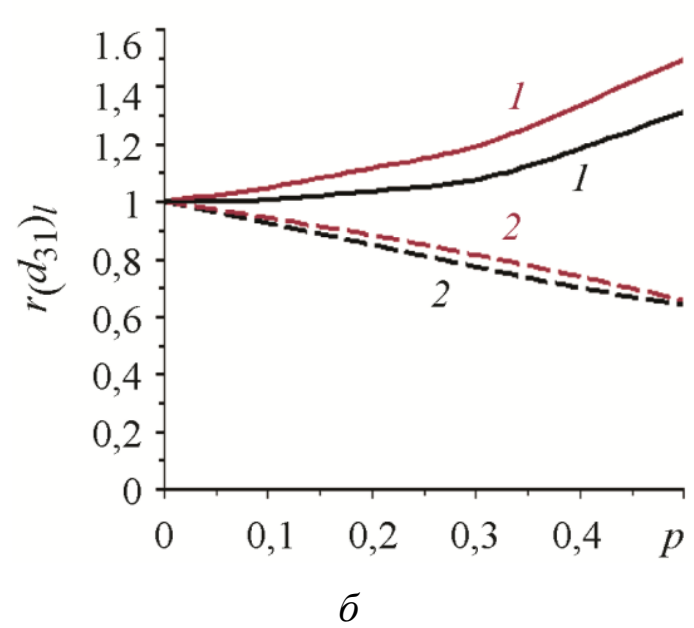

Рис. 5. Зависимости эффективных пьезомодулей $r\left(d_{33}\right)_{l}(a)$ и $r\left(d_{31}\right)_{l}(б)$ от пористости

Для пьезомодуля $\left(d_{33}^{\text {eff }}\right)_{2}$ обычной пористой пьезокерамики известно его необычное свойство слабой зависимости или небольшого убывания от пористости, хотя значения пьезомодуля $\left(\left|d_{31}^{\text {eff }}\right|\right)_{2}$ убывают более существенно с ростом $p$. Эти свойства, как видно из кривых 2 на рис. 5, слабо зависят от учета или не учета неоднородности поляризации.

Для пористой пьезокерамики с металлизированными границами пор, как видно из рис. 5 , значения пьезомодуля $\left(\left|d_{31}^{\text {eff }}\right|\right)_{1}$ возрастают с ростом $p$, причем учет неоднородности поля поляризации приводит к меньшему росту по абсолютной величине пьезомодуля $\left(\left|d_{31}^{\text {eff }}\right|\right)_{1}$. Пьезомодуль $\left(d_{33}^{\text {eff }}\right)_{1}$ при учете неоднородности поля поляризации практически ведет себя почти так же, как и для обычной пористой пьезокерамики, т.е. слабо убывает с ростом пористости. Если же считать пьезокерамический материал композита однородно поляризованным, то пьезомодуль $\left(d_{33}^{\text {eff }}\right)$ б будет даже возрастать с ростом пористости. Таким образом, можно сделать вывод, что учет неоднородности поляризации пьезокерамического материала композита с частично металлизированными поверхностями пор оказывает существенное влияние на величины пьезомодулей $d_{3 j}^{\text {eff }}$.

\section{6. Заключение}

В настоящей работе методами механики композитов и компьютерного моделирования исследованы свойства неоднородно поляризованной пористой пьезокерамики с частично металлизированными поверхностями пор. Металлизация пор учитывалась только электрическими граничными условиями эквипотенциальности. Результаты вычислительных экспериментов показали, что микропористая пьезокерамика с металлизированными поверхностями пор имеет ряд необычных свойств, перспективных для практических применений [39], т. е. эффективные модули диэлектрических проницаемостей пористой пьезокерамики с металлизированными поверхностями пор возрастают при увеличении пористости, тогда как для обычной пористой пьезокерамики эффективные модули диэлектрических проницаемостей убывают с ростом пористости. Наиболее интересным является увеличение с ростом пористости абсолютных значений $\left|e_{31}^{\text {eff }}\right|$ и $\left|d_{31}^{\text {eff }}\right|$ поперечных пьезомодулей, что также не наблюдается для обычной пористой пьезокерамики. При этом продольные пьезомодули $e_{33}^{\text {eff }}$ и $d_{33}^{\text {eff }}$ при учете металлизации изменяются не так существенно. 
Сравнение полученных результатов с аналогичными, приведенными в [31-35] для случая полной металлизации поверхностей пор, свидетельствует, что частичная металлизация немного нивелирует необычные свойства эффективных модулей $[32,34]$. Результаты расчетов также показали, что учет неоднородности поляризации пьезокерамического материала композита более существенен для определения эффективных значений пьезомодулей и диэлектрических проницаемостей, особенно для модулей с индексами, соответствующими основной оси поляризации, и менее важен для определения значений эффективных модулей жесткости.

Разработанная модель представительного объема обладает только частичнослучайной структурой пористости, поскольку имеются проходящие через весь объем области слои, не содержащие пор. В связи с этим, некоторые из отмеченных закономерностей влияния неоднородности поля поляризации могут измениться при других внутренних структурах представительных объемов. Также, на величины эффективных модулей оказывают влияние степень металлизации границ пор и толщина металлизированных покрытий, что было отмечено в [30-36] для случая однородно поляризованной пьезокерамики. Наконец, в случае рассмотрения композитов с наноразмерными порами необходимо учитывать размерные эффекты, например описываемые теориями поверхностных напряжений и их обобщений [40].

\section{Благодарность}

Работа выполнена при поддержке Российского фонда фундаментальных исследований, проект № 16-58-48009 Инд-оми, и Минобрнауки РФ, проект № 9.5070.2017/6.7, для первого автора.

\section{Литература}

1. Mercadelli E., Sanson A., Galassi C. Porous piezoelectric ceramics // Piezoelectric Ceramics / educated by E. Suaste-Gomez. - InTechOpen, 2010. - P. 111-128. - DOI: 10.5772/9942.

2. Development of porous piezoceramics for medical and sensor applications / E. Ringgaard, F. Lautzenhiser, L. M. Bierregaard, T. Zawada, E. Molz // Materials. - 2015. - Vol. 8, no. 12. P. 8877-8889. - DOI: 10.3390/ma8125498.

3. Rybyanets A. N. Porous piezoceramics: theory, technology, and properties // IEEE Trans. Ultrason. Ferroelectr. Freq. Control. - 2011. - Vol. 58. - P. 1492-1507. DOI: 10.1109/TUFFC.2011.1968.

4. Topolov V. Yu., Bowen C. R. Electromechanical Properties in Composites Based on Ferroelectrics. - London : Springer, 2009. - 202 p. - DOI: 10.1007/978-1-84882-000-5.

5. Piezoelectric and mechanical properties of $\mathrm{CaO}$ reinforced porous PZT ceramics with onedimensional pore channels / W. Liu, W. Liu, Y. Wang, C. Xue, J. Wang, J. Yang // Ceramics International. - 2017. - Vol. 43. - P. 2063-2068. - DOI: 10.1016/j.ceramint.2016.10.181.

6. Microstructure, electrical and mechanical properties of $\mathrm{MgO}$ nanoparticles-reinforced porous PZT 95/5 ferroelectric ceramics / J. Wang, H. Nie, C. Lan, G. Wang, X. Dong, X. Chen, F. Cao, H. He // Ceramics International. - 2013. - Vol. 39. - P. 3915-3919. DOI: 10.1016/j.ceramint.2012.10.238.

7. Mechanical and electrical properties of small amount of oxides reinforced PZT ceramics / P.-H. Xiang, X.-L. Dong, H. Chen, Z. Zhang, J.-K. Guo // Ceramics International. - 2003. Vol. 29. - P. 499-503. - DOI: 10.1016/S0272-8842(02)00193-1.

8. Rybyanets A. N., Naumenko A. A. Nanoparticles transport in ceramic matrixes: a novel approach for ceramic matrix composites fabrication // Journal of Modern Physics. - 2013. - Vol. 4, no. 8. - P. 1041-1049. - DOI: 10.4236/jmp.2013.48140.

9. ОСТ 11 0444-87. Материалы пьезокерамические. Технические условия. - 1980. $142 \mathrm{c}$. 
10. ANSI/IEEE Std. 176-1987: IEEE Standard on Piezoelectricity. - New York : IEEE, 1987. $66 \mathrm{p}$.

11. Processing and properties of porous piezoelectric materials with high hydrostatic figures of merit / C. R. Bowen, A. Perry, A. C. F. Lewis, H. Kara // J. Eur. Ceram. Soc. - 2004. - Vol. 24. P. 541-545. - DOI: 10.1016/S0955-2219(03)00194-8.

12. Fabrication and evaluation of porous piezoelectric ceramics and porosity-graded piezoelectric actuators / J. F. Li, K. Takagi, , M. Ono, W. Pan, R. Watanabe, A. Almajid, M. Taya // J. Am. Ceram. Soc. - 2003. - Vol. 86. - P. 1094-1098. - DOI: 10.1111/j.11512916.2003.tb03430.x.

13. Effects of foam composition on the microstructure and piezoelectric properties of macroporous PZT ceramics from ultrastable particle-stabilized foams / W. Liu, L. Du, Y. Wang, J. Yang, H. Xu // Ceramics International. - 2013. - Vol. 39. - P. 8781-8787. DOI: 10.1016/j.ceramint.2013.04.065.

14. Microstructure and electrical properties of porous PZT ceramics fabricated by different methods / A. K. Yang, C. A. Wang, R. Guo, Y. Huang // J. Am. Ceram. Soc. - 2010. - Vol. 93. P. 1984-1990. - DOI: 10.1111/j.1551-2916.2010.03684.x.

15. Getman I., Lopatin S. Theoretical and experimental investigation of the porous PZT ceramics // Ferroelectrics. - 1996. - Vol. 186. - P. 301-304. - DOI: 10.1080/00150199608218088.

16. Piezoelectric properties of the porous PZT and the porous PZT composite with silicone rubber / K. Hikita, K. Yamada, M. Nishioka, M. Ono // Ferroelectrics. - 1983. - Vol. 49. - P. 265-272. DOI: $10.1080 / 00150198308244698$.

17. Bowen C. R., Kara H. Pore anisotropy in 3-3 piezoelectric composites // Materials Chemistry and Physics. - 2002. - Vol. 75. - P. 45-49. - DOI: 10.1016/S0254-0584(02)00028-7.

18. Dunn H., Taya M. Micromechanics predictions of the effective electroelastic moduli of piezoelectric composites // Int. J. Solids Struct. - 1993. - Vol. 30. - P. 161-175. - DOI: 10.1016/00207683(93)90058-F.

19. Iyer S., Alkhader M., Venkatesh T.A. On the relationships between cellular structure, deformation modes and electromechanical properties of piezoelectric cellular solids // Int. J. Solids Struct. - 2016. - Vol. 80. - P. 73-83. - DOI: 10.1016/j.ijsolstr.2015.10.024.

20. Iyer S., Venkatesh T. A. Electromechanical response of (3-0, 3-1) particulate, fibrous, and porous piezoelectric composites with anisotropic constituents: A model based on the homogenization method // Int. J. Solids Struct. - 2014. - Vol. 51. - P. 1221-1234. DOI: 10.1016/j.ijsolstr.2013.12.008.

21. Microstructural modelling of the polarization and properties of porous ferroelectrics / R. W. C. Lewis, A. C. E. Dent, R. Stevens, C. R. Bowen // Smart Mater. Struct. - 2011 . - Vol. 20. P. 085002. - DOI: 10.1088/0964-1726/20/8/085002.

22. Homogenization of porous piezoelectric materials / G. Martinez-Ayuso, M. I. Friswell, S. Adhikari, H. H. Khodaparast, H. Berger // Int. J. Solids Struct. - 2017. - Vol. 113-114. P. 218-229. - DOI: 10.1016/j.ijsolstr.2017.03.003.

23. Наседкин А. В., Шевцова М. С. Моделирование эффективных модулей для различных типов пористых пьезокерамических материалов // Вестник ДГТУ. - 2013. - № 3-4 (72-73). C. 16-26.

24. Nasedkin A. V., Shevtsova M. S. Multiscale computer simulation of piezoelectric devices with elements from porous piezoceramics // Physics and Mechanics of New Materials and their Applications / ed. by I. A. Parinov and S.-H. Chang. - New York : Nova Science Publishers, 2013. - Ch. 16. - P. 185-202.

25. Effects of porosity distribution and porosity volume fraction on the electromechanical properties of 3-3 piezoelectric foams / B. V. Nguyen, K. S. Challagulla, T. A. Venkatesh, D. A. Hadjiloizi, A. V. Georgiades // Smart Mater. Struct. - 2016. - Vol. 25. - P. 125028. DOI: $10.1088 / 0964-1726 / 25 / 12 / 125028$. 
26. Паньков А. А. Статистическая механика пьезокомпозитов. - Пермь : Изд-во Перм. гос. техн. ун-та, 2009. - 480 с.

27. Perry A., Bowen C. R., Mahon S. W. Finite element modelling of 3-3 piezocomposites // Scripta Materialia. - 1999. - Vol.41, no. 9. - P. 1001-1007. - DOI: 10.1016/S13596462(99)00249-3.

28. Соколкин Ю. В., Паньков А. А. Электроупругость пьезокомпозитов с нерегулярными структурами. - М. : Физматлит, 2003. - 176 с.

29. Хорошун Л. Н., Маслов Б. П., Лещенко П. В. Прогнозирование эффективных свойств пьезоактивных композитных материалов. - Киев : Наукова Думка, 1989. - 347 с.

30. Наседкин А. В., Наседкина А. А., Рыбянец А. Н. Конечно-элементный анализ эффективных свойств микропористой пьезокерамики с частичной металлизацией поверхностей пор, учитываемой граничными условиями свободных электродов // Фундаментальные проблемы радиоэлектронного приборостроения. - 2017. - Т. 17, № 2. C. 336-339.

31. Наседкин А. В., Наседкина А. А., Рыбянец А. Н. О моделях микропористых пьезоэлектрических композитов, полученных методом транспорта металлосодержащих микрочастиц // Современные проблемы механики сплошной среды : труды XVIII международной конференции, г. Ростов-на-Дону, 7-10 ноября 2016 г. - Ростов-на-Дону : Изд-во ЮФУ, 2016. - T. 2. - C. 122-126.

32. Наседкин А. В., Наседкина А. А., Рыбянец А. Н. Численное исследование влияния жесткости металлизации поверхности пор на свойства микропористой пьезокерамики, полученной методом транспорта металлосодержащих частиц // Фундаментальные проблемы радиоэлектронного приборостроения. - 2016. - Т. 16, № 2. - С. 58-61.

33. Nasedkin A. V. Models and finite element approximations for interacting nanosized piezoelectric bodies and acoustic medium // AIP Conference Proceedings. - 2017. - Vol. 1798. P. 020181-1-020181-7. - DOI: 10.1063/1.4975643.

34. Nasedkin A., Nasedkina A., Rybyanets A. Finite element simulation of effective properties of microporous piezoceramic material with metallized pore surfaces // Ferroelectrics. - 2017. Vol. 508. - P. 100-107. - DOI: 10.1080/00150193.2017.1289569.

35. Nasedkin A. V., Nasedkina A. A., Rybyanets A. N. Mathematical modeling and computer design of piezoceramic materials with random arrangement of micropores and metallized pore surfaces // Proceedings of the 2016 International Conference on "Physics, Mechanics of New Materials and Their Applications" / ed. by I. A. Parinov, S.-H. Chang, M. A. Jani. - New York : Nova Science Publishers, 2017. - Ch. 54. - P. 385-392.

36. Nasedkin A. V., Nasedkina A. A., Rybyanets A. N. Modeling and computer design of piezoceramic materials with stochastic microporous structure and local alloying pore surfaces // Poromechanics VI : proceedings of the Sixth Biot Conference on Poromechanics, July 9-13, 2017, Paris, France / ed. by M. Vandamme, P. Dangla, J.-M. Pereiram, S. Ghabezloo. - Reston, Virginia, USA : ASCE, 2017. - P. 724-731. - DOI: 10.1061/9780784480779.089.

37. Nan C. W., Weng G. J. Influence of polarization orientation on the effective properties of piezoelectric composites // J. Appl. Phys. - 2000. - Vol. 88, no. 1. - P. 416-423. DOI: $10.1063 / 1.373675$.

38. Лехницкий С. Г. Теория упругости анизотропного тела. - М. : Наука, 1977. $416 \mathrm{c}$.

39. Nasedkin A. V., Nasedkina A. A., Rajagopal A. Finite Element Analysis of Cymbal Transducer from Porous Piezoceramics PZT-4 with Various Material Properties // Advanced Materials. PHENMA 2017 : proceedings of the International Conference on Physics and Mechanics of New Materials and Their Applications / ed. by I. A. Parinov, S.-H. Chang, V. K. Gupta. - Ser. Springer Proceedings in Physics. - Vol. 207. - Heidelberg, New York, Dordrecht, London : Springer Cham, 2018. - DOI: 10.1007/978-3-319-78919-4_42. 
40. Wang K. F., Wang B. L., Kitamura T. A review on the application of modified continuum models in modeling and simulation of nanostructures // Acta Mech. Sin. - 2016 - Vol. 32, no 1. P. 83-100. - DOI: 10.1007/s1040-9-015-0508-4. 